\title{
Anomalous Magnetic Moments and Quark Orbital Angular Momentum
}

\author{
Matthias Burkardt \\ Department of Physics, New Mexico State University, Las Cruces, NM 88003-0001, U.S.A. \\ Gunar Schnell \\ Subatomaire en Stralingsfysica, Universiteit Gent, 9000 Gent, Belgium
}

(Dated: February 16, 2007)

\begin{abstract}
We derive an inequality for the distribution of quarks with nonzero orbital angular momentum, and thus demonstrate, in a model-independent way, that a nonvanishing anomalous magnetic moment requires both a nonzero size of the target as well as the presence of wave function components with quark orbital angular momentum $L_{z}^{q}>0$.

PACS numbers: 13.40.Em,13.60.-r,13.88.+e,14.20.-c
\end{abstract}

\section{INTRODUCTION}

Detailed measurements of the spin structure of the nucleon 1, 2, 3, 4, 5, 6, 7, 8, 9, 10, 11 have revealed that only a small fraction of the nucleon spin is carried by the quark spin. This result immediately raised the question, which degrees of freedom carry the rest. Unfortunately, both orbital angular momentum and the gluon spin are difficult to access experimentally, and therefore little rigorous information exists about quark orbital angular momentum.

Meanwhile, many qualitative statements regarding orbital angular momentum have been made. For example, when one expresses the matrix element for the anomalous magnetic moment of the nucleon in terms of light-cone wave functions (summed over all Fock components), a nonzero anomalous magnetic moment can only result when there is a nonzero probability that the vector current flips the nucleon helicity [12, 13]. Since the same matrix element conserves the spin of the quarks it is evident that some orbital angular momentum must be transfered to the quarks. Hence a nonzero anomalous magnetic moment can only occur when the target wave function contains components with nonzero orbital angular momentum. While this argument is rigorous, it leaves open quantitative questions regarding the norm of those wave function components or perhaps the resulting net $L_{z}^{q}$. Within models, it has also been found that a point-like object cannot produce a nonzero anomalous magnetic moment [12, 14] and within this model one can even derive quantitative bounds.

Similarly, the observation of a nonzero Sivers [15] effect by the HERMES collaboration [16] seems to indicate wavefunction components with nonzero $L_{z}^{q}$ since the effect requires an interference between initial nucleon states that have opposite helicity. Furthermore, orbital angular momentum seems to play a central role in all models for the Sivers function [17, 18, 19, 20, 21].

The main purpose of this note is to make some of the statements regarding the anomalous magnetic moment more quantitative. In order to accomplish this goal, we start from the matrix element that yields the generalized parton distribution $E^{q}$ and apply the Cauchy-Schwarz inequality which will then provide a lower bound on the norm of wave function components with nonzero orbital angular momentum.

\section{DECOMPOSITION OF THE NUCLEON SPIN}

In $\mathrm{QCD}$, there is no unique way to decompose the nucleon spin into quark spin, quark angular momentum, gluon spin, and gluon orbital angular momentum. For example, Ji has considered a decomposition based on the $M^{012}$ component of the angular momentum tensor 22]

$$
M^{012}=\frac{i}{2} q^{\dagger}(\vec{r} \times \vec{D})^{z} q+\frac{1}{2} q^{\dagger} \sigma^{z} q+2 \operatorname{Tr} E^{j}(\vec{r} \times \vec{D})^{z} A^{j}+\operatorname{Tr}(\vec{E} \times \vec{A})^{z} .
$$

The matrix elements of the four terms in Eq. (11) are interpreted as the quark orbital angular momentum, quark spin, gluon orbital angular momentum, and gluon spin respectively. One advantage of this decomposition is gauge invariance, another is the fact that the matrix elements of the first term in Eq. (1) can be probed in deeply virtual Compton scattering. A disadvantage of this decomposition is that the orbital angular momenta in Eq. (11) contain interactions through the gauge covariant derivative.

An alternative decomposition of the total angular momentum starts from $M^{+12}$ in light cone gauge $A^{+} \equiv A^{0}+A^{z}=$ 
$0[23]$

$$
M^{+12}=\frac{1}{2} q_{+}^{\dagger}(\vec{r} \times i \vec{\partial})^{z} q_{+}+\frac{1}{2} q_{+}^{\dagger} \gamma_{5} q_{+}+2 \operatorname{Tr} F^{+j}(\vec{r} \times i \vec{\partial})^{z} A^{j}+\varepsilon^{+-i j} \operatorname{Tr} F^{+i} A^{j}
$$

Here $q_{+} \equiv \frac{1}{2} \gamma^{-} \gamma^{+} q$ is the dynamical component of the quark field operators. The obvious disadvantage of using Eq. (2) to interpret the total angular momentum of the nucleon, which we all know is equal to $\frac{1}{2} \hbar$, as a sum of quark and gluon orbital and spin angular momentum, respectively, is the fact that Eq. (2) is not gauge invariant. However, it is invariant under the residual group of gauge transformations that leave $A^{+}=0$ and where the $A^{j}$ satisfy antisymmetric boundary conditions at $x^{-}= \pm \infty$. The main advantages of using Eq. (2) to decompose the nucleon spin is the fact that all terms in Eq. (2) are quadratic in the fields. Unlike Eq. (11) there is therefore no ambiguity as to the interpretation of interaction terms. Another advantage is the fact that the matrix elements of the various terms in Eq. (2) can be easily expressed in terms of light-cone wave functions.

We should emphasize that although we have listed here some of the most obvious advantages and disadvantages of these two possibilities for decomposing the angular momentum, we do not consider one or the other superior in general. However, for specific applications one of these two decompositions may be advantageous. In particular, if one wants to place constraints on light-cone wave functions, which find many applications in hadron phenomenology, then one may prefer the decomposition of the orbital angular momentum based on $M^{+12}$ (2). In the rest of this paper we will exclusively study the light-cone decomposition of $J_{z}$, based on Eq. (2).

We thus consider in the following the orbital angular momentum of quarks with flavor $q$

$$
L_{z}^{q}=\int d x^{-} \int d^{2} \mathbf{x}_{\perp} \frac{1}{2} q_{+}^{\dagger}(\vec{r} \times i \vec{\partial})^{z} q_{+}
$$

and we will investigate its role in nucleon spin-flip matrix elements, such as the anomalous magnetic moment.

As it stands, Eq. (3) is gauge dependent. We therefore need to specify the gauge. First of all, we impose the light-cone gauge condition $A^{+}=0$. However, this does not yet completely fix the gauge since $A^{+}$remains zero under gauge transformations with a phase that only depends on $\mathbf{x}_{\perp}$. We thus impose as an additional condition that the $\perp$ component of the gauge field satisfies anti-periodic boundary conditions at light-cone infinity

$$
\mathbf{A}_{\perp}\left(x^{-}=\infty, \mathbf{x}_{\perp}\right)=-\mathbf{A}_{\perp}\left(x^{-}=-\infty, \mathbf{x}_{\perp}\right) .
$$

With this additional condition, the definition (3) of $L_{z}^{q}$ becomes unique.

Although all results that we will be deriving will be valid in this gauge only, we believe they will nevertheless be useful since a lot of hadron phenomenology is based on light-cone wave functions and their use usually implies the use of light-cone gauge. It is therefore fair to say that although our results will not have a gauge-independent interpretation, they will nevertheless provide additional insights about light-cone wave functions of hadrons.

\section{ANOMALOUS MAGNETIC MOMENT AND GPDS}

We first consider the generalized parton distribution $E\left(x, 0,-\boldsymbol{\Delta}_{\perp}^{2}\right)$, which appears in non-forward nucleon spin-flip matrix elements of light-cone correlation functions. For purely transverse momentum transfer $p^{+}=p^{\prime+}$ one finds

$$
\begin{aligned}
& \left\langle P+\Delta, \uparrow\left|O^{q}\left(x, \mathbf{0}_{\perp}\right)\right| P, \uparrow\right\rangle=H^{q}\left(x, 0,-\Delta_{\perp}^{2}\right) \\
& \left\langle P+\Delta, \uparrow\left|O^{q}\left(x, \mathbf{0}_{\perp}\right)\right| P, \downarrow\right\rangle=-\frac{\Delta_{x}-i \Delta_{y}}{2 M} E^{q}\left(x, 0,-\Delta_{\perp}^{2}\right)
\end{aligned}
$$

where

$$
O^{q}\left(x, \mathbf{b}_{\perp}\right)=\int \frac{d x^{-}}{4 \pi} e^{i p^{+} x^{-} x} \bar{q}\left(0^{-}, \mathbf{b}_{\perp}\right) \gamma^{+} q\left(x^{-}, \mathbf{b}_{\perp}\right)
$$

(in gauges other than light-cone gauge $A^{+}=0$ one needs to insert a Wilson line gauge link in Eq. (7)). In the forward limit, integration over $x$ yields the anomalous magnetic moment contribution from quarks with flavor $q$

$$
\int d x E^{q}(x, 0,0)=\kappa^{q} .
$$

Since the operator in Eq. (6) is chirally even, the matrix element is diagonal in quark spin. Since the matrix element involves a nucleon spin-flip, angular momentum conservation thus requires a change in quark orbital angular 
momentum. As a result, the mere fact that the anomalous magnetic moment of the nucleon is nonzero implies that there must be components in the nucleon wave function that have a nonzero orbital angular momentum [13] (of course, this does not necessarily mean that there is any net orbital angular momentum). In the following we will try to make this statement more quantitative, i.e., we will attempt to place constraints on the probability to find nonzero orbital angular momentum components in the nucleon wave function.

In order to facilitate the separation between intrinsic orbital angular momentum and the orbital angular momentum due to the motion of the entire nucleon, we first switch to a representation of states that are eigenstates of the transverse center of momentum

$$
\left|p^{+}, \mathbf{R}_{\perp}, \lambda\right\rangle \equiv \mathcal{N} \int d^{2} \mathbf{p}_{\perp}\left|p^{+}, \mathbf{p}_{\perp}, \lambda\right\rangle e^{i \mathbf{b}_{\perp} \cdot \mathbf{R}_{\perp}},
$$

where $\mathcal{N}$ is a normalization constant. In this basis, we can define impact parameter dependent parton distributions as

$$
\begin{aligned}
\mathcal{H}^{q}\left(x, \mathbf{b}_{\perp}\right) & =\left\langle p^{+}, \mathbf{R}_{\perp}, \uparrow\left|O^{q}\left(x, \mathbf{b}_{\perp}\right)\right| p^{+}, \mathbf{R}_{\perp}, \uparrow\right\rangle \\
\left(\frac{\partial}{\partial b_{x}}-i \frac{\partial}{\partial b_{y}}\right) \frac{\mathcal{E}^{q}\left(x, \mathbf{b}_{\perp}\right)}{2 M} & =\left\langle p^{+}, \mathbf{R}_{\perp}, \uparrow\left|O^{q}\left(x, \mathbf{b}_{\perp}\right)\right| p^{+}, \mathbf{R}_{\perp}, \downarrow\right\rangle .
\end{aligned}
$$

The impact parameter dependent PDFs are related to GPDs via a simple Fourier transform 24, 25, 26, 27]

$$
\begin{aligned}
\mathcal{H}\left(x, \mathbf{b}_{\perp}\right) & =\int \frac{d^{2} \boldsymbol{\Delta}_{\perp}}{(2 \pi)^{2}} e^{i \mathbf{b}_{\perp} \cdot \boldsymbol{\Delta}_{\perp}} H\left(x, 0,-\boldsymbol{\Delta}_{\perp}^{2}\right) \\
\mathcal{E}\left(x, \mathbf{b}_{\perp}\right) & =\int \frac{d^{2} \boldsymbol{\Delta}_{\perp}}{(2 \pi)^{2}} e^{i \mathbf{b}_{\perp} \cdot \boldsymbol{\Delta}_{\perp}} E\left(x, 0,-\boldsymbol{\Delta}_{\perp}^{2}\right)
\end{aligned}
$$

and the normalization is such that $\int d^{2} \mathbf{b}_{\perp} q\left(x, \mathbf{b}_{\perp}\right)=q(x)$.

Since the GPDs provide simultaneous information about the longitudinal momentum and the transverse position of partons, it is instructive to introduce creation operators in this "hybrid space". For example, for $x>0$ we define destruction operators for quarks with momentum fraction $x$ at transverse position $\mathbf{b}_{\perp}$

$$
b\left(x p^{+}, \mathbf{b}_{\perp}\right)=\int d x^{-} e^{i p+x^{-} x} q_{+}\left(x^{-}, \mathbf{b}_{\perp}\right)
$$

and similarly for anti-particles $(x<0)$. For now we will suppress the helicity of the quarks and consider only quantities that have been summed over the helicities of the quarks. In terms of these, the impact parameter dependent PDFs take on a particularly simple form $(x>0)$

$$
\begin{aligned}
\mathcal{H}\left(x, \mathbf{b}_{\perp}\right) & =\left\langle p^{+}, \mathbf{R}_{\perp}, \uparrow\left|b^{\dagger}\left(x p^{+}, \mathbf{b}_{\perp}\right) b\left(x p^{+}, \mathbf{b}_{\perp}\right)\right| p^{+}, \mathbf{R}_{\perp}, \uparrow\right\rangle \\
\frac{1}{2 M}\left(\frac{\partial}{\partial b_{x}}+i \frac{\partial}{\partial b_{y}}\right) \mathcal{E}\left(x, \mathbf{b}_{\perp}\right) & =\left\langle p^{+}, \mathbf{R}_{\perp}, \uparrow\left|b^{\dagger}\left(x p^{+}, \mathbf{b}_{\perp}\right) b\left(x p^{+}, \mathbf{b}_{\perp}\right)\right| p^{+}, \mathbf{R}_{\perp}, \downarrow\right\rangle,
\end{aligned}
$$

which emphasizes their physical interpretation as densities. Upon introducing

$$
B_{+}^{q}(x) \equiv \int d^{2} \mathbf{b}_{\perp}\left(b_{x}+i b_{y}\right) b^{\dagger}\left(x p^{+}, \mathbf{b}_{\perp}\right) b\left(x p^{+}, \mathbf{b}_{\perp}\right)
$$

and integration by parts we find

$$
\left\langle p^{+}, \mathbf{R}_{\perp}, \uparrow\left|B_{+}^{q}(x)\right| p^{+}, \mathbf{R}_{\perp}, \downarrow\right\rangle=\frac{1}{2 M} E^{q}(x, 0,0) .
$$

We can use this result to provide a formal proof that $\Delta L_{z}^{q}=1$ in the matrix element defining $E^{q}(x, 0,0)$. Indeed, one easily verifies the commutation relation

$$
\left[L_{z}^{q}, B_{+}^{q}(x)\right]=B_{+}^{q}(x),
$$

which proves that $B_{+}^{q}$ has nonvanishing matrix elements only between states that differ by one unit of orbital angular momentum $L_{z}^{q}$ for flavor $q$. This observation is consistent with results based on overlap integrals of light-cone wave functions [13]. 


\section{ANGULAR MOMENTUM DECOMPOSITION OF PDFS}

In order to derive some quantitative constraints on the light-cone wave functions of hadrons, we first introduce an angular momentum decomposition for parton distributions, i.e.,

$$
b^{\dagger}\left(x p^{+}, \mathbf{b}_{\perp}\right)=\sum_{m} b_{m}^{\dagger}\left(x p^{+}, \mathbf{b}_{\perp}\right)
$$

where

$$
b_{m}^{\dagger}\left(x p^{+}, \mathbf{b}_{\perp}\right)=e^{+i m \phi_{b}} \int_{0}^{2 \pi} \frac{d \phi_{b}^{\prime}}{2 \pi} e^{-i m \phi_{b}^{\prime}} b^{\dagger}\left(x p^{+}, \mathbf{b}_{\perp}^{\prime}\right)
$$

where $b_{x}=\left|\mathbf{b}_{\perp}\right| \cos \phi$ and $b_{y}=\left|\mathbf{b}_{\perp}\right| \sin \phi$, and $b_{x}^{\prime}=\left|\mathbf{b}_{\perp}\right| \cos \phi_{b}^{\prime}$ and $b_{y}^{\prime}=\left|\mathbf{b}_{\perp}\right| \sin \phi_{b}^{\prime}$, respectively.

Of course, the above decomposition into orbital angular momentum components is scale dependent due to the scale dependence of light-cone wave functions [28]. However, we imagine performing such a decomposition at a fixed scale at which we perform the analysis of this decomposition.

The physical interpretation of the creation and destruction operators (21) is that they create quarks with $m$ units of angular momentum (in the z-direction), i.e.,

$$
\left[L_{z}^{q}, b_{m}^{\dagger}\left(x p^{+}, \mathbf{b}_{\perp}\right)\right]=m b_{m}^{\dagger}\left(x p^{+}, \mathbf{b}_{\perp}\right) .
$$

In terms of these angular momentum projected creation operators, the operator $B_{+}^{q}$ appearing in the impact parameter space representation of the matrix element for the anomalous magnetic moment takes on the form

$$
B_{+}^{q}=\sum_{m} \int d^{2} \mathbf{b}_{\perp}\left(b_{x}+i b_{y}\right) b_{m+1}^{\dagger}\left(x p^{+}, \mathbf{b}_{\perp}\right) b_{m}\left(x p^{+}, \mathbf{b}_{\perp}\right) .
$$

Inserting the angular mode expansion into Eq. (18) and applying the Cauchy-Schwarz inequality to scalar products between states yields

$$
\begin{aligned}
\frac{E^{q}(x, 0,0)}{2 M} \leq & \sum_{m} \int d^{2} \mathbf{b}_{\perp}\left(b_{x}+i b_{y}\right)\left\langle p^{+}, \mathbf{0}_{\perp}, \uparrow\left|b_{m+1}^{\dagger}\left(x p^{+}, \mathbf{b}_{\perp}\right) b_{m}\left(x p^{+}, \mathbf{b}_{\perp}\right)\right| p^{+}, \mathbf{0}_{\perp}, \downarrow\right\rangle \\
\leq \sum_{m} \int d^{2} \mathbf{b}_{\perp}\left|\mathbf{b}_{\perp}\right| & \sqrt{\left\langle p^{+}, \mathbf{0}_{\perp}, \uparrow\left|b_{m+1}^{\dagger}\left(x p^{+}, \mathbf{b}_{\perp}\right) b_{m+1}\left(x p^{+}, \mathbf{b}_{\perp}\right)\right| p^{+}, \mathbf{0}_{\perp}, \uparrow\right\rangle} \\
& \times \sqrt{\left\langle p^{+}, \mathbf{0}_{\perp}, \downarrow\left|b_{m}^{\dagger}\left(x p^{+}, \mathbf{b}_{\perp}\right) b_{m}\left(x p^{+}, \mathbf{b}_{\perp}\right)\right| p^{+}, \mathbf{0}_{\perp}, \downarrow\right\rangle} .
\end{aligned}
$$

In order to simplify the notation, we now introduce the distribution of partons with orbital angular momentum $m$ in a target with spin $\uparrow$

$$
q_{m}^{\uparrow}(x) \equiv \int d^{2} \mathbf{b}_{\perp}\left\langle p^{+}, \mathbf{o}_{\perp}, \uparrow\left|b_{m}^{\dagger}\left(x p^{+}, \mathbf{b}_{\perp}\right) b_{m}\left(x p^{+}, \mathbf{b}_{\perp}\right)\right| p^{+}, \mathbf{o}_{\perp}, \uparrow\right\rangle
$$

as well as the $\mathbf{b}_{\perp}^{2}$-weighted distribution of partons with orbital angular momentum $m$

$$
b_{m}^{2 \uparrow}(x) \equiv \int d^{2} \mathbf{b}_{\perp}\left\langle p^{+}, \mathbf{0}_{\perp}, \uparrow\left|b_{m}^{\dagger}\left(x p^{+}, \mathbf{b}_{\perp}\right) b_{m}\left(x p^{+}, \mathbf{b}_{\perp}\right)\right| p^{+}, \mathbf{0}_{\perp}, \uparrow\right\rangle \mathbf{b}_{\perp}^{2} .
$$

Obviously we have

$$
\begin{aligned}
q_{m}^{\downarrow}(x) & =q_{-m}^{\uparrow}(x) \\
b_{m}^{2, \downarrow}(x) & =b_{-m}^{2, \uparrow}(x) .
\end{aligned}
$$

To each term in the sum in Eq. (24) we now apply the Cauchy-Schwarz inequality for integrals

$$
\int d^{2} \mathbf{b}_{\perp} \sqrt{f\left(\mathbf{b}_{\perp}\right) g\left(\mathbf{b}_{\perp}\right)} \leq \sqrt{\int d^{2} \mathbf{b}_{\perp} f\left(\mathbf{b}_{\perp}\right)} \sqrt{\int d^{2} \mathbf{b}_{\perp} g\left(\mathbf{b}_{\perp}\right)} .
$$


For $m \geq 0$ we apply this inequality with

$$
\begin{aligned}
f\left(\mathbf{b}_{\perp}\right) & =\int d^{2} \mathbf{b}_{\perp}\left\langle p^{+}, \mathbf{0}_{\perp}, \uparrow\left|b_{m+1}^{\dagger}\left(x p^{+}, \mathbf{b}_{\perp}\right) b_{m+1}\left(x p^{+}, \mathbf{b}_{\perp}\right)\right| p^{+}, \mathbf{0}_{\perp}, \uparrow\right\rangle=q_{m+1}^{\uparrow}(x) \\
g\left(\mathbf{b}_{\perp}\right) & =\int d^{2} \mathbf{b}_{\perp} \mathbf{b}_{\perp}^{2}\left\langle p^{+}, \mathbf{o}_{\perp}, \downarrow\left|b_{m}^{\dagger}\left(x p^{+}, \mathbf{b}_{\perp}\right) b_{m}\left(x p^{+}, \mathbf{b}_{\perp}\right)\right| p^{+}, \mathbf{0}_{\perp}, \downarrow\right\rangle=b_{m}^{2, \downarrow}(x),
\end{aligned}
$$

while for $m<0$ we identify

$$
\begin{aligned}
f\left(\mathbf{b}_{\perp}\right) & =\int d^{2} \mathbf{b}_{\perp} \mathbf{b}_{\perp}^{2}\left\langle p^{+}, \mathbf{0}_{\perp}, \uparrow\left|b_{m+1}^{\dagger}\left(x p^{+}, \mathbf{b}_{\perp}\right) b_{m+1}\left(x p^{+}, \mathbf{b}_{\perp}\right)\right| p^{+}, \mathbf{0}_{\perp}, \uparrow\right\rangle=b_{m+1}^{2 \uparrow}(x) \\
g\left(\mathbf{b}_{\perp}\right) & =\int d^{2} \mathbf{b}_{\perp}\left\langle p^{+}, \mathbf{0}_{\perp}, \downarrow\left|b_{m}^{\dagger}\left(x p^{+}, \mathbf{b}_{\perp}\right) b_{m}\left(x p^{+}, \mathbf{b}_{\perp}\right)\right| p^{+}, \mathbf{o}_{\perp}, \downarrow\right\rangle=q_{m}^{\downarrow}(x),
\end{aligned}
$$

yielding

$$
\begin{aligned}
\frac{E^{q}(x, 0,0)}{2 M} & \leq \sum_{m \geq 0} \sqrt{q_{m+1}^{\uparrow}(x) b_{m}^{2, \downarrow}(x)}+\sum_{m<0} \sqrt{b_{m+1}^{2, \uparrow}(x) q_{m}^{\downarrow}(x)} \\
& =\sum_{m \geq 0} \sqrt{q_{m+1}^{\uparrow}(x) b_{-m}^{2 \uparrow}(x)}+\sum_{m<0} \sqrt{b_{m+1}^{2 \uparrow}(x) q_{-m}^{\uparrow}(x)} \\
& =\sum_{m \geq 0} \sqrt{q_{m+1}^{\uparrow}(x) b_{-m}^{2, \uparrow}(x)}+\sum_{m>0} \sqrt{q_{m}^{\uparrow}(x) b_{1-m}^{2, \uparrow}(x)} \\
& =2 \sum_{m \geq 0} \sqrt{q_{m+1}^{\uparrow}(x) b_{-m}^{2, \uparrow}(x)} \\
& \leq 2 \sqrt{\left(\sum_{m \geq 0} q_{m+1}^{\uparrow}(x)\right)\left(\sum_{n \geq 0} b_{-n}^{2, \uparrow}(x)\right)}
\end{aligned}
$$

where in the last step we used $\left(f_{m}, g_{m} \geq 0\right)$

$$
\sum_{m} \sqrt{f_{m} g_{m}} \leq \sqrt{\left(\sum_{m} f_{m}\right)\left(\sum_{n} g_{n}\right)}
$$

If we now introduce the distribution of quarks with positive angular momenta in a nucleon with spin in the $+\hat{z}$ direction

$$
q_{L_{z} \geq 1}(x)=\sum_{m \geq 1} q_{m}^{\uparrow}(x)
$$

as well as the contribution to $\mathbf{b}_{\perp}^{2}(x)$ from quarks with zero or negative $L_{z}^{q}$

$$
b_{L_{z} \leq 0}^{2}(x)=\sum_{m \leq 0} b_{m}^{2, \uparrow}(x)
$$

our result can be cast into the form

$$
\left(\frac{E^{q}(x, 0,0)}{4 M}\right)^{2} \leq q_{L_{z} \geq 1}(x) b_{L_{z} \leq 0}^{2}(x),
$$

which is the main result of this paper. A slightly stronger inequality can be obtained by keeping track of the quark helicity. For this purpose we note that both quark helicities contribute equally in Eq. (18). Upon repeating the above analysis starting from quarks with helicity $\uparrow$, one thus arrives at

$$
\frac{E^{q}(x, 0,0)}{4 M} \leq \sqrt{q_{L_{z} \geq 1, \uparrow}^{\uparrow}(x) b_{L_{z} \leq 0, \downarrow}^{2, \uparrow}(x)}+\sqrt{q_{L_{z} \geq 1, \downarrow}^{\uparrow}(x) b_{L_{z} \leq 0, \uparrow}^{2, \uparrow}(x)},
$$

where for example $q_{L_{z} \geq 1, \downarrow}^{\uparrow}(x)$ denotes the distribution of quarks with helicity $\downarrow$ and positive orbital angular momentum in a nucleon with helicity $\uparrow$. An even stronger version of Eq. (38) can be achieved following Ref. [29] by starting with $\gamma^{+}\left(1 \pm \gamma_{5}\right) / 2$ instead of only $\gamma^{+}$in Eq. (7). In that case only one of the two terms on the r.h.s. of (38) remains, and $\frac{E}{4 M}$ gets replaced by $\frac{E}{8 M}[30]$. Additional simplified inequalities can be derived if one neglects quarks with orbital angular momenta $\left|L_{z}^{q}\right| \geq 2$. 


\section{DISCUSSION}

First of all, our result illustrates that a nonvanishing anomalous magnetic moment (and hence nonvanishing $E(x, 0,0))$ implies both

- wave function components with positive quark orbital angular momentum (for $S_{z}=+\frac{1}{2}$ )

- a nonvanishing $\perp$ size

The fact that the size of hadrons needs to be nonzero and that nonzero angular momentum components need to be present if a state has an anomalous magnetic moment has been observed before in light-cone wave function models of hadrons [13, 14]. What is new in our paper is the fact that our result provides a model independent, quantitative lower bound for the $\perp$ size distribution, which unlike in Ref. 29] is connected to the quark orbital angular momentum. Furthermore, we have shown that there must be quarks with orbital angular momentum in the same direction as the nucleon spin - regardless of the sign of the anomalous magnetic moment. This is at first surprising, since one might have expected that if the anomalous magnetic moment is due to an orbital angular momentum then, when $E(x, 0,0)<0$ the quark should orbit opposite to the nucleon spin. However, one needs to keep in mind that we make no statement about the net orbital angular momentum. We only derived a lower bound on contributions to the net $L_{z}^{q}$ from modes with $L_{z}^{q}>0$.

The necessity of quark orbital angular momentum for an anomalous magnetic moment is particularly surprising from the nonrelativistic quark model (NRQM) point of view. In the NRQM the anomalous magnetic moment is entirely due to the spins of the quarks and no orbital angular momentum is needed. However, in this point the NRQM is not completely consistent: For example, when a massive Dirac particle is in some bound state then, due to the localization, the particle must necessarily possess a nonzero momentum and thus a nonvanishing lower Dirac component. The lower component for a massive s-wave quark has orbital angular momentum $L_{z}^{q}=1$. Since even the $d$ quark appears in the NRQM wave functions with both spin up and down, this relativistic effect gives rise to wave function components with both positive and negative orbital angular momentum. In our inequality (37), the lower bound on orbital angular momentum is proportional to the inverse radius squared of the quark distribution. Strictly speaking the non-relativistic limit requires $R m_{q} \rightarrow \infty$ and hence $R M \rightarrow \infty$. In this limit the lower bound on $q_{L_{z} \geq 1}$ goes to zero. Therefore, in a system where the nonrelativistic approximation would be allowed, a nonzero anomalous magnetic moment would not necessarily require nonzero orbital angular momentum.

What is also surprising is that, once $E(x, 0,0)$ is nonzero, there need to be quarks with positive orbital angular momentum - regardless of the sign of $E(x, 0,0)$. This is because for $E(x, 0,0)$ to be nonzero there ought to be a nonzero matrix element between a nucleon with spin up and a nucleon with spin down in Eq. (18). For example, if the active quark in the initial state had $L_{z}^{q}=0$, then it needs to have $L_{z}^{q}=-1$ in the final state, i.e., $L_{z}^{q}$ in the same direction as the nucleon spin. Likewise if $L_{z}^{q}=0$ in the final state, $L_{z}^{q}$ must have been +1 in the initial state in Eq. (18). Similarly one can convince oneself in the general case (initial and final state have nonzero $L_{z}^{q}$ ) that a nonzero $E(x, 0,0)$ always requires wave function components with $L_{z}^{q}>1$ in a nucleon that has $S_{z}=\uparrow$. This is surprising since in Ji's relation the sign of $E$ seems to suggest the sign of $L_{z}^{q}$. However, one needs to keep in mind that Ji's relation deals with the net $L_{z}^{q}$ and secondly the angular momentum decomposition to which the Ji relation applies (10) does not have to be the same as the angular momentum decomposition in the light-cone framework (2).

In order to get some quantitative feeling for the lower bound, we consider a model for $u$-quarks in the valence region where $(\kappa=2)$

$$
\begin{aligned}
q(x) & =8(1-x)^{3} \\
E(x, 0,0) & =6 \kappa(1-x)^{5}=12(1-x)^{5} \\
M^{2} b^{2}(x) & =M^{2} R^{2} 8(1-x)^{5}
\end{aligned}
$$

where we pick $R=0.5 \mathrm{fm}$ for the transverse size, i.e., $M R=2.5$ and we let $b^{2}(x) \propto(1-x)^{2} q(x)$, consistent with a finite size for large $x$. Since no data is available on $E(x, 0,0)$ and $b^{2}(x)$, we simply make an educated guess regarding these functions in order to be able to make an order of magnitude estimate for the probability to find nonzero orbital angular momentum. The ansatz for $E(x, 0,0)$ is motivated by the constraint that $E(x, 0,0)$ must vanish by two powers of $(1-x)$ faster than $q(x)$ for large $x$. The ansatz for $b^{2}(x)$ was motivated by the constraint that the nucleon has a finite transverse size for large $x$. The rest of the above ansatz was guided by the ad hoc requirement to make the model as simple as possible and therefore the idea is that this model should merely serve as a guide for what order of magnitude one should expect ("back of an envelope estimate") with parameters that are within the range of what is expected in the valence region. With the above model parameters we find for the distribution of quarks with positive orbital angular momentum

$$
\frac{9}{50}(1-x)^{5} \leq q_{L_{z} \geq 1}(x)
$$


i.e., only some \% compared to the distribution summed over all orbital angular momenta.

While this is much smaller than current estimates based on Ji's relation (see for example Ref. 31] for an up to date estimate), one should keep in mind that the orbital angular momentum obtained from the Ji relation differs from the orbital angular momentum from the light-cone decomposition (2).

Although our lower bound is not very spectacular, it still provides the first model-independent lower bound on the distribution of quarks with positive angular momentum distribution. One may also wonder how such a low bound is consistent with transverse flavor dipole moments $\left|d_{y}^{q}\right| \approx 0.2 \mathrm{fm}$ for a moving nucleon that are quite significant and almost the same order of magnitude as the $\perp$ size of the nucleon. The important point here is that the $\perp$ distortion, which is described by our starting equation $E$, contains a piece involving the overlap between the $L_{z}^{q}=0$ component of the wave function and the $L_{z}^{q}=1$ component, i.e., it is linear in the amplitude for finding a quark with nonzero orbital angular momentum, whereas the probability for $L_{z}^{q}>0$ is quadratic in that amplitude. The situation is thus somewhat analogous to the quadrupole moment of the deuteron, which is linear in the d-wave component and quite large, while the actual d-wave probability is tiny.

\section{SUMMARY}

We have derived an inequality, which provides a model-independent lower bound on the norm of wave-function components with nonzero quark orbital angular momentum. Although the numerical bound thus obtained is not very strong — requiring only a few \% probability for nonzero orbital angular momentum - our result represents the first quantitative estimate for wave-function components with nonzero $L_{z}^{q}$. Moreover, the resulting constraints on the light-cone wave functions of the nucleon, which only enter the distributions quadratically, are much stronger. The bound that we derive involves the transverse size (rms-radius) times the mass of the nucleon. This quantifies the known result that a point-like particle cannot have a nonzero anomalous magnetic moment. Unlike $L_{z}^{q}$ itself, which depends quadratically on wave-function components with nonzero $L_{z}^{q}$, the $\operatorname{GPD} E(x, 0,0)$ requires only that $L_{z}^{q}$ is nonzero on one side of the matrix element. This illustrates why we were only able to derive a bound on the probability for nonzero $L_{z}^{q}$ that depends quadratically on $E(x, 0,0)$, which is why our bound is so weak. In contradistinction $L_{z}^{q}$ obtained through the Ji relation would involve $E(x, 0,0)$ linearly. Nevertheless, we hope that the constraints derived in this work will be of use in developing better models for light-cone wave functions describing these interesting observables.

Acknowledgments: We would like to thank M. Diehl, M. Oka and W. Vogelsang for stimulating discussions. M.B. was partially supported by the DOE under grant number DE-FG03-95ER40965. M.B. would also like to thank T.-A. Shibata for his hospitality and support during part of this project. G.S. was partially supported by the Japan Society for the Promotion of Science, the Alexander-von-Humboldt Stiftung, and the Fonds voor Wetenschappelijk Onderzoek - Vlaanderen.

[1] J. Ashman et al. (EMC), Phys. Lett. B206, 364 (1988).

[2] D. Adams et al. (SMC), Phys. Rev. D56, 5330 (1997), hep-ex/9702005.

[3] D. Adams et al. (SMC), Phys. Lett. B396, 338 (1997).

[4] B. Adeva et al. (SMC), Phys. Rev. D58, 112001 (1998).

[5] B. Adeva et al. (SMC), Phys. Rev. D60, 072004 (1999).

[6] E. S. Ageev et al. (COMPASS), Phys. Lett. B612, 154 (2005), hep-ex/0501073.

[7] K. Abe et al. (E143), Phys. Rev. D58, 112003 (1998), hep-ph/9802357.

[8] P. L. Anthony et al. (E155), Phys. Lett. B463, 339 (1999), hep-ex/9904002.

[9] P. L. Anthony et al. (E155), Phys. Lett. B493, 19 (2000), hep-ph/0007248.

[10] A. Airapetian et al. (HERMES), Phys. Lett. B442, 484 (1998).

[11] X. Zheng et al. (Jefferson Lab Hall A), Phys. Rev. C70, 065207 (2004), nucl-ex/0405006.

[12] S. J. Brodsky and S. D. Drell, Phys. Rev. D22, 2236 (1980).

[13] S. J. Brodsky, D. S. Hwang, B.-Q. Ma, and I. Schmidt, Nucl. Phys. B593, 311 (2001), hep-th/0003082.

[14] S. J. Brodsky and F. Schlumpf, Phys. Lett. B329, 111 (1994), hep-ph/9402214.

[15] D. W. Sivers, Phys. Rev. D43, 261 (1991).

[16] A. Airapetian et al. (HERMES), Phys. Rev. Lett. 94, 012002 (2005), hep-ex/0408013.

[17] C. Boros, Z. T. Liang, and T. C. Meng, Phys. Rev. Lett. 70, 1751 (1993).

[18] S. J. Brodsky, D. S. Hwang, and I. Schmidt, Phys. Lett. B530, 99 (2002), hep-ph/0201296.

[19] M. Burkardt, Nucl. Phys. A735, 185 (2004), hep-ph/0302144.

[20] F. Yuan, Phys. Lett. B575, 45 (2003), hep-ph/0308157.

[21] A. Bacchetta, A. Schaefer, and J.-J. Yang, Phys. Lett. B578, 109 (2004), hep-ph/0309246. 
[22] X. Ji, Phys. Rev. Lett. 78, 610 (1997), hep-ph/9603249.

[23] R. L. Jaffe and A. Manohar, Nucl. Phys. B337, 509 (1990).

[24] M. Burkardt, Phys. Rev. D62, 071503 (2000), hep-ph/0005108.

[25] M. Burkardt, Int. J. Mod. Phys. A18, 173 (2003), hep-ph/0207047.

[26] M. Diehl, Eur. Phys. J. C25, 223 (2002), hep-ph/0205208.

[27] A. V. Belitsky, X. Ji, and F. Yuan, Phys. Rev. D69, 074014 (2004), hep-ph/0307383.

[28] M. Burkardt, X. Ji, and F. Yuan, Phys. Lett. B545, 345 (2002), hep-ph/0205272.

[29] M. Burkardt, Phys. Lett. B582, 151 (2004), hep-ph/0309116.

[30] M. Diehl, private communications.

[31] M. Diehl (2005), hep-ph/0510221. 